\title{
Rapid identification and typing of Yersinia pestis and other Yersinia species by matrix-assisted laser desorption/ionization time-of-flight (MALDI-TOF) mass spectrometry
}

\author{
Saravanan Ayyadurai, Christophe Flaudrops, Didier Raoult, Michel Drancourt ${ }^{*}$
}

\begin{abstract}
Background: Accurate identification is necessary to discriminate harmless environmental Yersinia species from the food-borne pathogens Yersinia enterocolitica and Yersinia pseudotuberculosis and from the group A bioterrorism plague agent Yersinia pestis. In order to circumvent the limitations of current phenotypic and PCR-based identification methods, we aimed to assess the usefulness of matrix-assisted laser desorption/ionization time-offlight (MALDI-TOF) protein profiling for accurate and rapid identification of Yersinia species. As a first step, we built a database of 39 different Yersinia strains representing 12 different Yersinia species, including 13 Y. pestis isolates representative of the Antiqua, Medievalis and Orientalis biotypes. The organisms were deposited on the MALDI-TOF plate after appropriate ethanol-based inactivation, and a protein profile was obtained within 6 minutes for each of the Yersinia species.
\end{abstract}

Results: When compared with a 3,025-profile database, every Yersinia species yielded a unique protein profile and was unambiguously identified. In the second step of analysis, environmental and clinical isolates of $Y$. pestis $(n=2)$ and Y. enterocolitica $(n=11)$ were compared to the database and correctly identified. In particular, $Y$. pestis was unambiguously identified at the species level, and MALDI-TOF was able to successfully differentiate the three biotypes.

Conclusion: These data indicate that MALDI-TOF can be used as a rapid and accurate first-line method for the identification of Yersinia isolates.

\section{Background}

The Yersinia genus is comprised of 15 species of $\gamma$-proteobacteria (http://www.bacterio.cict.fr/xz/yersinia.html) that are mostly harmless environmental organisms residing in soil and water [1]. Three Yersinia species are human pathogens, including Yersinia pseudotuberculosis, Yersinia enterocolitica and the plague agent Yersinia pestis [2-4]. While the two former species are food-borne pathogens responsible primarily for enteric infections, $Y$. pestis is an ectoparasite-borne species responsible for deadly plague [2]. Moreover, $Y$. pestis has been classified in the Centers for Disease Control's (CDC's) group A list

\footnotetext{
* Correspondence: michel.drancourt@univmed.fr

Unité de Recherche sur les Maladies Infectieuses et Tropicales Emergentes: URMITE, UMR CNRS 6236-IRD 198, Faculté de Médecine, IFR48, Université de la Méditerranée, Marseille, France
}

(c) 2010 Ayyadurai et al; licensee BioMed Central Ltd. This is an Open Access article distributed under the terms of the Creative Commons Attribution License (http://creativecommons.org/licenses/by/2.0), which permits unrestricted use, distribution, and reproduction in any medium, provided the original work is properly cited. of potential bioterrorism agents (http://www.bt.cdc.gov/ agent/agentlist-category.asp). Thus, rapid and accurate methods of detection and identification are needed for the distinction of $Y$. pestis among other Yersinia species, as well as Yersinia organisms among other Enterobacteriaceae species.

Conventional methods for the phenotypic identification of Yersinia organisms such as biochemical profiling are time-consuming: they require the manipulation of huge quantities of potentially harmful pathogens and delay accurate identification beyond an appropriate time limit with respect to the medical management of patients and public health issues. PCR-based techniques [5] and real-time PCR assays reduce these delays to a few hours but require expertise and expensive reagents [6]. Furthermore, due to the natural instability of 
$Y$. pestis plasmids and chromosomal regions, molecular analysis may lead to false negative results when targeting specific genomic regions such as the 3 a signature sequence [7-9]. Recognition of the F1 capsular antigen by several immunological techniques has been used for the rapid detection and identification of $Y$. pestis collected from patients with suspected infections [10] and from skeleton specimens from historical plague burial sites [11].

The identification of bacteria by matrix-assisted laser desorption/ionization time-of-flight (MALDI-TOF) mass spectrometry (MS) has recently emerged as a rapid and sensitive technology that provides protein profiles for the accurate identification of bacteria at the genus, species or sub-species level $[12,13]$. In microbiology, MALDI-TOF-MS has a number of potential advantages over other typing methods. Specimen preparation is relatively simple and can be carried out within minutes. Furthermore, the technique does not require any taxonspecific or costly materials such as antibodies. The workflow is simple and fast and can be standardized for most bacterial species. In addition, many of the procedures for sample preparation, data acquisition, and evaluation can be automated. Although MALDI-TOF-MS has been applied to several Enterobacteriaceae species, including $Y$. enterocolitica [14], it has not been described for other pathogenic Yersinia species, and only one report has dealt with the avirulent $Y$. pestis vaccinal strain EV 76 [15]. In this study, we assessed whether MALDI-TOF-MS could accurately identify all Yersinia species, including the three major biotypes of $Y$. pestis.

\section{Methods}

\section{Bacterial strains}

The following isolates were used to create an updated MALDI-TOF database comprising of 12 Yersinia species, except for Yersinia similis, Yersinia aleksiciae and Yersinia entomophaga: Yersinia pestis 6/69M strain Orientalis biotype (kindly provided by Michel Simonet, Institut Pasteur, Lille, France), $Y$. pestis Nairobi-rattus (Antiqua biotype), $Y$. pestis 14-47 strain Medievalis biotype (kindly provided by Joseph B. Hinnebusch, Rocky Mountain Laboratory, Hamilton, Montana and Florent Sebbane, Institut Pasteur, Lille, France), Y. pestis EV 76 (vaccine strain), six $Y$. pestis Medievalis isolates (5F1, 6b4, 8B7, 9F1, 5G5, 5B9) [16], Y. enterocolitica subsp. enterolitica CIP 8027, Y. enterolitica subsp. paleartica CIP 106945, Y. enterocolitica subsp. enterocolitica CIP 106676 (serotype 0:3), Y. enterocolitica subsp. enterocolitica CIP 8142 (serotype 0:9), Y. enterocolitica subsp. enterocolitica CIP 101776, Y. pseudotuberculosis CIP 5585, Y. frederiksenii CIP 8029, Y. intermedia CIP 8028, Y. kristensenii CIP 8030, Y. bercovieri CIP 103323,
Y. mollaretii CIP 103324, Y. rohdei CIP 103163, Y. ruckeri CIP 8280, Y. aldovae CIP 103162, and Y. massiliensis CIP $109351^{\mathrm{T}}$ [17]. To test the identification abilities of MALDI-TOF, we used additional environmental and clinical isolates, including $Y$. pestis JHUPRI strain [18], two $Y$. pestis Orientalis biotype strains recently isolated from rodents in Algeria [19], ten $Y$. enterocolitica serotype O:9 (biotype 2) clinical isolates from feces in Nigeria (in collaboration with Joseph AE Okwori, Federal College of Veterinary and Medical Laboratory Technology, National Veterinary Research Institute, Vom, Nigeria), and one $Y$. enterocolitica strain isolated in our laboratory from stool. According to the French law, informed consent is not required from the individuals as far as the study concerns only microbiota and not the individuals themselves. The study of these isolates was approved by the Ethics Committee, Institute Fédératif de Recherche 48, Marseille, France. The Yersinia isolates were cultured on trypticase soy agar plates at $28^{\circ} \mathrm{C}$ for 2 days, and all $Y$. pestis isolates were cultured in a P3 laboratory in a biosafety level III cabinet with appropriate confinement protocols. Strains were identified by partial PCR amplification and sequencing of the rров gene [20]. Y. pestis typing was performed by multispacer sequencing typing (MST) using the spacers YP1, YP3, YP4, YP5, YP7, YP8, YP9, and YP10 as previously described [21]. The presence of plasmids in the $Y$. pestis isolates was evaluated by PCR analysis of plasmids pPst (pPCP1) [22], pFra (pMT1) [23] and pCD1 (pYV) via amplification of a 659-bp fragment of the $\operatorname{lcr} V$ gene using the forward primer 5'-GAATTGGTTCAGTTAGTCAA-3' and reverse primer 5'-AGATTACCCAACGCCCCGGT-3' and the following PCR program: an initial denaturation for $5 \mathrm{~min}$ at $95^{\circ} \mathrm{C}$ and 39 cycles of denaturation for $1 \mathrm{~min}$ at $94^{\circ} \mathrm{C}$, annealing for $30 \mathrm{~s}$ at $56^{\circ} \mathrm{C}$ and extension for $1 \mathrm{~min}$ at $74^{\circ} \mathrm{C}$, followed by a final extension for $10 \mathrm{~min}$ at $74^{\circ} \mathrm{C}$. PCR products were analyzed in a $1.5 \%$ agarose gel containing $2 \mu \mathrm{g} / \mathrm{ml}$ ethidium bromide.

\section{Protocol for the inactivation of Yersinia organisms}

To inactivate the bacteria, a $10-\mu \mathrm{l}$ volume of $70 \%$ ethanol was added to the bacterial growth, vortexed in a biosafety level III cabinet and incubated at room temperature for $1 \mathrm{~h}$. The effectiveness of the inactivation protocol for all samples was assayed prior to MALDITOF analysis by inoculating $50 \mu \mathrm{l}$ of inactivated Yersinia suspension on a $5 \%$ sheep-blood agar plate and $50 \mu \mathrm{l}$ into trypticase soy broth (AES, Rennes, France) and incubated them in parallel at $28^{\circ} \mathrm{C}$ for 7 days. The absence of any visible growth after 7 days of incubation was taken as evidence that the inactivation protocol was effective. 


\section{MALDI-TOF-MS database}

For each inactivated isolate, we deposited $1.5 \mu$ of this suspension covered with $1.5 \mu \mathrm{l}$ of matrix solution [saturated solution of alpha-cyano-4-hydroxycinnamic acid $(\alpha-\mathrm{HCCA})$ in $50 \%$ acetonitrile, $2.5 \%$ trifluoracetic acid] on a TP 384 target plate made of polished steel T F (Bruker Daltonics, Leipzig, Germany) and the matrix was then air-dried for 5 minutes. MALDI-TOF measurements were carried out using an Autoflex II mass spectrometer (Bruker Daltonics, Wissembourg, France) equipped with a 337-nm nitrogen laser. The instrument was calibrated every day using a reference Klebsiella pneumoniae isolate. Spectra were recorded in the positive linear mode (delay, $170 \mathrm{~ns}$; ion source 1 (IS1) voltage, $20 \mathrm{kV}$; ion source 2 (IS2) voltage, $18.5 \mathrm{kV}$; lens voltage, $7 \mathrm{kV}$; mass range, $2-20 \mathrm{kDa}$ ). For each Yersinia sp. strain, the whole cell's protein profile was determined in triplicate. Each spectrum was obtained after 675 shots in automatic mode at variable laser power, and the time of acquisition was 30-60 seconds per spot. Automated data acquisition was performed with AutoXecute acquisition control software. The raw spectra obtained for each isolate were imported into MALDI BioTyper $^{\mathrm{Tu}}$ version 2.0 software (Bruker Daltonics) and analyzed by standard pattern matching (with default parameter settings) against the MALDI BioTyper ${ }^{\mathrm{ru}}$ database, an integrated part of the software (June 2008 version). Proteins between 3-15 kDa were identified by their $\mathrm{m} / \mathrm{z}$ values. For each spectrum, up to 100 peaks were considered and compared to peaks in the database. The results were visualized with an intuitive graphical user interface. The peaks that were most similar (mass difference $<600 \mathrm{ppm}$ ) to the reference spectra appeared in green, while peaks with a mass difference $>600 \mathrm{ppm}$ were shown in red or yellow. The 12 bacterial species exhibiting the most similar protein pattern to the strain under study were ranked by an identification score. The database (commercially available at Bruker Daltonics) was comprised of 3,025 MALDI-TOF profiles, including 42 strains of 11 Yersinia species, but lacking $Y$. pestis. We incorporated the profiles that we obtained for 39 different Yersinia isolates representative of 12 Yersinia species, including $13 \mathrm{Y}$. pestis strains, into this database. Every Yersina strain profile obtained in this study was also copied to a separate folder to form a new database in addition to the MALDI BioTyper ${ }^{\text {ru }}$ database. The profiles were matched with the existing MALDI BioTyper ${ }^{\mathrm{m}}$ database, and identification of the bacteria was carried out using MALDI BioTyper ${ }^{\text {Tim }}$ version 2.0.

\section{MALDI-TOF-MS identification}

A total of 13 Yersinia isolates including 2 environmental $Y$. pestis Orientalis biotype isolates and 11 clinical isolates of $Y$. enterocolitica collected from feces were inactivated and blindly analyzed by MALDI-TOF-MS against the local updated database as described above. Identification scores were assigned using the following scoring parameters [13]: a score $\geq 1.9$ indicated species identification; a score of 1.7-1.9 indicated genus identification; and a score $<1.7$ indicated no identification. An isolate was considered to be correctly identified by MALDI-TOF when two of two spectra had a score $\geq 1$.9. For organisms identified as $Y$. pestis, we further separated the protein profiles into three folders corresponding to each of the three biotypes. Using ClinPro Tools software, we analyzed the specific protein profile pattern for each biotype. ClinPro Tools software inbuild, quick classifier and genetic algorithm analyses were used to differentiate the three $Y$. pestis biotypes. Quick classifier compares the average sprectum of the differentes classes in order to find the specific different peaks. The genetic algorithm creates a random peak list, changes the list ("mutation") and compares the discriminating capacity until obtaining the best list for discriminating classes.

\section{Reproducibility of MALDI-TOF-MS identification}

In order to assess the reproducibility of MALDI-TOFMS identification, every strain studied was tested in triplicate (i.e., on three different MALDI-TOF plates run on three different days from three different batches of culture). For every condition, 4 different spots were loaded on the MALDI-TOF plate, giving a total of 12 MALDI-TOF-MS protein profiles that were derived from each strain.

\section{Results \\ Constituting a MALDI-TOF-MS Yersinia database}

Accurate identification at the species level was confirmed for every isolate by partial sequencing of the rpoB gene. In addition, the presence of $Y$. pestis was confirmed by sequencing specific targets in each plasmid for each of the $Y$. pestis isolates used in this study. MST analysis discriminated the $13 \mathrm{Y}$. pestis isolates into 3 biotypes (Antiqua, Midievalis and Orientalis) with smaller variation in the number of alleles than previously reported [21]. The MST profile for the $Y$. pestis JHUPRI strain was most closely related to the Antiqua biotype but was atypical in that it contained spacer sequences from each of the three biotypes. The original MST profiles were deposited at the following website: http:// ifr48.timone.univ-mrs.fr/MST_YPestis/mst. We observed no growth over 7 days for any of the $Y$. pestis isolates being studied after ethanol inactivation. MALDI-TOF protein profiles for the three main biotypes following $70 \%$ ethanol inactivation, including $Y$. pestis Antiqua (Y. pestis Nairobi-rattus), Medievalis ( $Y$. pestis 14-47), and Orientalis $(Y$. pestis $6 / 69 \mathrm{M})$ are shown in Figure 1. 


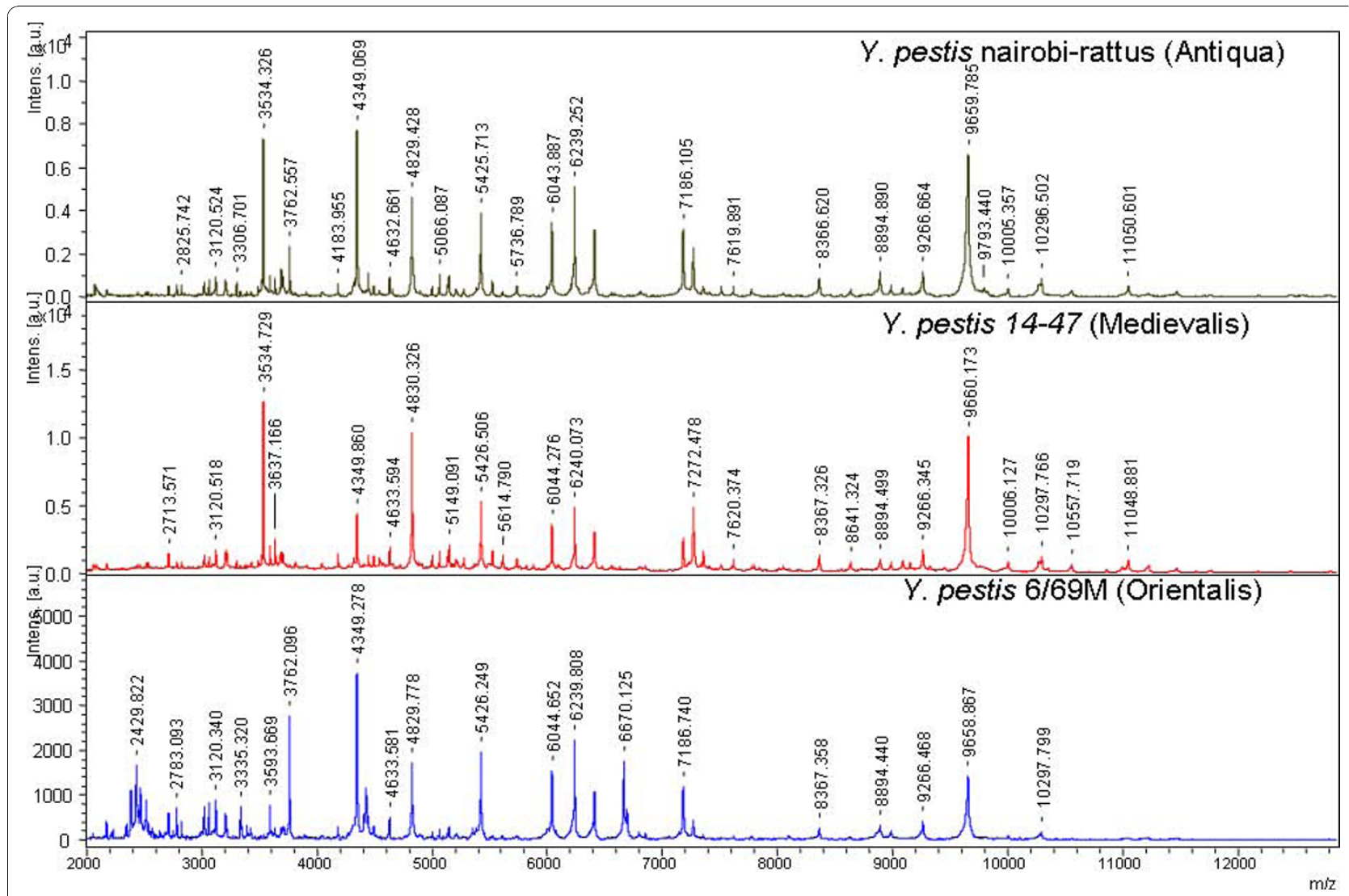

Figure 1 Protein profile of the major Y. pestis biotypes generated by MALDI-TOF-MS. a.i., arbitrary intensity given by the software.

Figure 2 contains a pseudo-gel representing the protein profile for the three $Y$. pestis biotypes.

\section{MALDI-TOF-MS identification of Yersinia organisms}

For the $Y$. pestis isolates, default identification against the Bruker database resulted in a false result of $Y$. pseudotuberculosis with an identification score $>2$ in two of two cases. When the identification was performed using our local updated database, the isolates were correctly matched as $Y$. pestis in two of two cases with an identification score $>2.7$, effectively identifying the isolates at the species level. The $11 Y$. enterocolitica isolates were correctly identified as $Y$. enterocolitica with an identification score $>2$. Further analysis of the $Y$. pestis isolates using ClinPro Tools software allowed us to assign them to a biotype, with the exception of the $Y$. pestis JHUPRI strain for which the unique MALDI-TOF profile did not match any of the three biotypes.

\section{Reproducibility of MALDI-TOF-MS identification}

We obtained a unique MALDI-TOF profile for each of the 39 Yersinia isolates being studied: for each isolate, the 12 MALDI-TOF profiles derived from triplicate analysis were similar and yielded identical, accurate identification. A list of $\mathrm{m} / \mathrm{z}$ values characteristic for $Y$. pestis is given in additional file 1 .

\section{Discussion}

Given that the MALDI BioTyper ${ }^{\mathrm{TM}}$ database contained 42 Yersinia profiles derived from 11 species but lacked the major pathogen $Y$. pestis, as well as the recently described species $Y$. massiliensis [17], we aimed to complete this database by deriving a MALDI-TOF profile for 12 species currently included in the Yersinia genus [17]. We obtained a unique MALDI-TOF profile for each of the Yersinia species used in this study. In each case, the species-specific profile did not match any of the 3,000 non-Yersinia profiles deposited in the MALDI BioTyper $^{\mathrm{TM}}$ database, including those for closely-related enteric bacteria. We further observed that the MALDITOF profiles of Yersinia isolates yielded reproducible, accurate identification in agreement with previous reports indicating that varying culture conditions did not alter the accurate identification of enteric bacteria, including $Y$. enterocolitica [24,25]. We further established the proof of concept that MALDI-TOF-MS can be used for the identification of organisms belonging to any of the 12 species studied here. Blind MALDI-TOF 


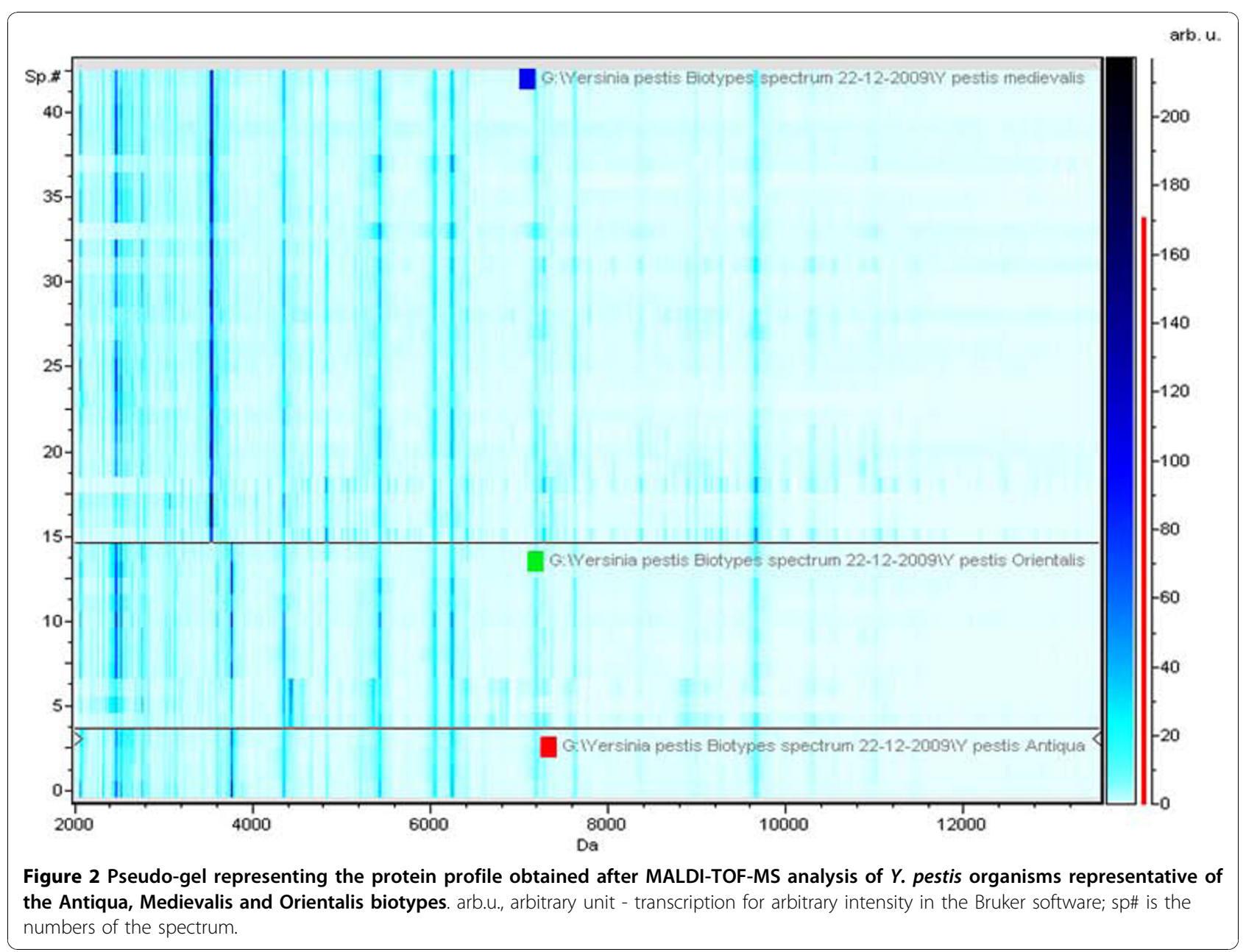

analysis yielded an identification score $\geq 2$ in 11 of 11 (100\%) clinical isolates of $Y$. enterocolitica and in 2 of 2 (100\%) of the $Y$. pestis isolates when compared to the updated database. An identification score $\geq 2$ has been described as a valuable cut-off point for the accurate identification of bacterial isolates by MALDI-TOF analysis [13]. The ability to correctly identify isolates blindly indicates that MALDI-TOF is indeed a new and effective method for Yersinia species identification. This had already been established for $Y$. enterocolitica organisms but had not been described for the other pathogenic Yersinia species as the only report on $Y$. pestis included just the avirulent vaccinal strain EV 76 [15]. Notably, updating the database was crucial for the accurate identification of isolates as MALDI-TOF analysis of $Y$. pestis isolates using the original Bruker database resulted in false identification as $Y$. pseudotuberculosis with an identification score $>2$. It has been previously observed that the quality of MALDI-TOF identification depends on the completeness and quality of the database used [13].
By using ClinPro Tools software as a second step, we were able to discriminate between the three main $Y$. pestis biotypes. The $Y$. pestis JHUPRI strain, however, was not identified as any of the three biotypes, in agreement with MST data indicating that it is an atypical strain [18]. This is consistent with previous observations that MALDI-TOF profiling is able to discriminate between various biotypes among other enteric species such as Salmonella enterica [26]. MALDI-TOF analysis can be supplemented with other state of the art techniques to ensure accurate genotyping of Yersinia isolates, including $Y$. pestis. While $16 \mathrm{~S}$ rDNA sequencing and rpoB gene sequencing yield accurate identification of Yersinia organisms at the species level, $[17,27,28]$ molecular typing of Yersinia organisms was done by MST [21], tandem repeat analysis [29-31], the detection of specific single-nucleotide polymorphisms [32], Enterobacterial Repetitive Intergenic Consensus PCR and Multilocus Sequence Analysis [27]. Mass spectrometry could be used for such determination thanks to emerging mass spectrometry-based methods for DNA analysis [33]. 
In this study, we inactivated all of the Yersinia organisms being studied even though such inactivation is not necessary for isolates belonging to species other than $Y$. pestis or when dealing with avirulent $Y$. pestis strains as previously reported [15]. We carried out an inactivation protocol to ensure that it did not significantly modify the results of the MALDI-TOF analysis. All of the inactivated Yersinia isolates with reference profiles in the Bruker database were unambiguously identified using this database, indicating that the inactivation protocol did not significantly interfere with MALDI-TOF identification. We hypothesized that a previously published inactivation protocol based on the incubation of $Y$. pestis with Tween and formalin, an agent that denatures proteins, may significantly modify the peptide profiles of isolates and affect their identification [33]. As expected, the inactivation of Yersinia by incubation with $80 \%$ TFA for 30 minutes as previously proposed for vegetative cells and spores did not yield interpretable profiles (data not shown) [34]. The protocols for ethanol inactivation tested in this study took 1 hour to inactivate the organisms; however, this step may be omitted if the mass spectrometer is used in a biosafety level 3 laboratory, although this was not the situation in our study. MALDI-TOF-MS identification can be completed in less than 10 minutes, less time than is required for Gram staining analysis [13]. The mass spectra of whole cells provide a snapshot of different protein compositions of individual microbial strains and thus constitute strainspecific suites of biomarkers. MALDI-TOF identification, therefore, is a more rapid technique for the identification of Yersinia isolates. Previously, only detection of the F1 capsular antigen using hand-held kits had proven to be an excellent bench-top technique for the rapid identification of $Y$. pestis [35]. In a comparative analysis, detection of the F1 antigen was highly specific and sensitive enough to positively identify ten of ten $Y$. pestis isolates from various countries [35]. The delay in identification varies from 20 minutes for an immunochromatographic test [10] to 2 hours for immunofluorescence microscopy [35]: however, the most accurate immunochromatographic test is not yet commercially available [35]. Given that it is based on the analysis of dozens of phenotypic characteristics into a unique profile, MALDI-TOF identification is less prone to variability and false negative results than phenotypic identification based on only one phenotypic characteristic such as the $Y$. pestis F1 capsular antigen. The F1 capsular antigen is plasmid-encoded and might be unstable; thus, it is risky to assume correct identification based on just one phenotypic trait. False negative results have been reported in cultures incubated at temperatures less than $37^{\circ} \mathrm{C}$ as this antigen is expressed by $Y$. pestis only between $33-37^{\circ} \mathrm{C}$ [1]. The same holds true with regard to direct detection of the F1 capsular antigen in specimens that have been refrigerated for more than 30 hours [1]. Therefore, MALDI-TOF identification appears to be the most rapid test for the accurate identification of $Y$. pestis and other Yersinia species organisms.

\section{Conclusion}

In conclusion, MALDI-TOF can be used as a first-line method for the accurate identification of Yersinia organisms using an updated database that includes profiles of all Yersinia species. MALDI-TOF identification is the most rapid identification tool and is available at a low cost $(<\$ 1$ per identification). $Y$. pestis should be added to the list of bioterrorism agents such as Bacillus anthracis that are readily identifiable by MALDI-TOFMS [36,37].

\section{Additional material}

Additional file 1: List of $\mathrm{m} / \mathrm{z}$ values of MALDI-TOF peaks characteristic for $Y$. pestis: $\mathrm{m} / \mathrm{z}$ values are given in the first column, the signal/noise $(\mathrm{S} / \mathrm{N})$ ratio is given in the second column.

\section{Acknowledgements}

The authors acknowledge Mr. Philippe Hoest for his help in handling $Y$. pestis isolates in the BSL3 laboratory.

\section{Authors' contributions}

AS, DR and MD designed the experiments and wrote the paper. AS and CF performed the experiments. DR and MD coordinated the project. All authors have read and approved the manuscript.

Received: 2 July 2010 Accepted: 12 November 2010

Published: 12 November 2010

\section{References}

1. Perry RD, Fetherston JD: Yersinia pestis - etiologic agent of plague. Clin Microbiol Rev 1997, 10:35-66.

2. Gage KL, Kosoy MY: Natural history of plague: perspectives from more than a century of research. Annu Rev Entomol 2005, 50:505-528.

3. Bottone EJ: Yersinia enterocolitica: overview and epidemiologic correlates. Microbes Infect 1999, 1:323-333.

4. Carniel E, Mollaret HH: Yersiniosis. Comp Immunol Microbiol Infect Dis 1990, 13:51-58.

5. Hinnebusch J, Schwan TG: New method for plague surveillance using polymerase chain reaction to detect Yersinia pestis in fleas. J Clin Microbiol 1993, 31:1511-1514.

6. Chase CJ, Ulrich MP, Wasieloski LP Jr, Kondig JP, Garrison J, Lindler LE, Kulesh DA: Real-time PCR assays targeting a unique chromosomal sequence of Yersinia pestis. Clin Chemist 2005, 51:1778-1785.

7. Wang X, Han Y, Li Y, Guo Z, Song Y, Tan Y, Du Z, Rakin A, Zhou D, Yang R: Yersinia genome diversity disclosed by Yersinia pestis genome-wide DNA microarray. Can J Microbiol 2007, 53:1211-1221.

8. Zhou D, Han Y, Dai E, Pei D, Song Y, Zhai J, Du Z, Wang J, Guo Z, Yang R: Identification of signature genes for rapid and specific characterization of Yersinia pestis. Microbiol Immunol 2004, 48:263-269.

9. Radnedge L, Chin SG, Mccready PM, Worsham PL, Andersen GL: Identification of nucleotide sequences for the specific and rapid detection of Yersinia pestis.. App Environ Microbial 2001, 67:3759-3762.

10. Chanteau S, Rahalison L, Ralafiarisoa L, Foulon J, Ratsitorahina M, Ratsifasoamanana L, Carniel E, Nato F: Development and testing of a rapid 
diagnostic test for bubonic and pneumonic plague. Lancet 2003, 361:211-216.

11. Bianucci R, Rahalison L, Peluso A, Massa MR, Ferroglio E, Signoli M, Langlois $J Y$, Gallien V: Plague immunodetection in remains of religious exhumed from burial sites in central France. J Archaeol Sci 2009, 36:616-621.

12. Sauer S, Freiwald A, Maier T, Kube M, Reinhardt R, Kostrzewa M, Geider K: Classification and identification of bacteria by mass spectrometry and computational analysis. PLOS ONE 2008, 3:e2843.

13. Seng P, Drancourt M, Gouriet F, La Scola B, Fournier PE, Rolain JM, Raoult D: Ongoing revolution in bacteriology: routine identification of bacteria by matrix-assisted laser desorption ionization time-of-flight mass spectrometry. Clin Infect Dis 2009, 49:543-551.

14. Parisi D, Magliulo M, Nanni P, Casale M, Forina M, Roda A: Analysis and classification of bacteria by matrix-assisted laser desorption/ionization time-of-flight mass spectrometry and a chemometric approach. Anal Bioanal Chem 2008, 391:2127-2134.

15. Liu H, Du Z, Wang J, Yang R: Universal sample preparation method for characterization of bacteria by Matrix-Assisted Laser Desorption Ionization - Time of Flight Mass Spectrometry. Appl Environ Microbiol 2007, 73:1899-1907.

16. Houhamdi L, Raoult D: Different genes govern Yersinia pestis pathogenicity in Caenorhabditis elegans and human lice. Microb Pathog 2008, 44:435-437.

17. Merhej V, Adékambi T, Pagnier I, Raoult D, Drancourt M: Yersinia massiliensis sp. nov., isolated from fresh water. Int J Syst Evol Microbiol 2008, 58:779-784.

18. Laforce FM, Acharya IL, Stott G, Brachman PS, Kaufman AF, Clapp RF, Shah NK: Clinical and epidemiological observations on an outbreak of plague in Nepal. Bull World Health Organ 1971, 45:693-706.

19. Bitam I, Ayyadurai S, Kernif T, Chetta M, Boulaghman N, Raoult D, Drancourt M: A new rural focus of Orientalis plague, Algeria. Emerg Infect Dis 2010.

20. Adékambi T, Drancourt M, Raoult D: rpoB gene as a tool for clinical microbiologist. Trends Microbiol 2009, 17:37-45.

21. Drancourt M, Roux V, La Vu D, Tran-Hung L, Castex D, Chenal-Francisque V, Ogata H, Fournier PE, Crubézy E, Raoult D: Genotyping, Orientalis-like Yersinia pestis, and plague pandemics. Emerg Infect Dis 2004, 10:1585-1592

22. Pouillot F, Derbise A, Kukkonen M, Foulon J, Korhonen TK, Carniel E: Evaluation of O-antigen inactivation on Pla activity and virulence of Yersinia pseudotuberculosis harbouring the pPla plasmid. Microbiology 2005, 151:3759-3768

23. Kuske CR, Barns SM, Grow CC, Merrill L, Dunbar J: Environmental survey for four pathogenic bacteria and closely related species using phylogenetic and functional genes. J Forensic Sci 2006, 51:548-558.

24. Wunschel DS, Hill EA, McLean JS, Jarman K, Gorby YA, Valentine N, Wahi K: Effect of varied $\mathrm{pH}$, growth rate and temperature using controlled fermentation and batch culture on Matrix Assisted Laser Desorption/ Ionization whole cell protein fingerprints. Journal Micobiol Methods 2005, 62:259-271.

25. Valentine N, Wunschel S, Wunschel S, Petersen C, Wahl K: Effect of culture conditions on microorganisms identification by Matrix-Assisted Laser Desorption Ionization Mass Spectrometry. Appl Environ Microbiol 2005, 71:58-64.

26. Lynn EC, Chung MC, Tsai WC, Han CC: Identification of Enterobacteriaceae bacteria by direct matrix-assisted laser desorptiom/ionization mass spectrometric analysis of whole cells. Rapid Commun Mass Spectrom 1999, 13:2022-2027.

27. Souza RA, Pitondo-Silva A, Falcão DP, Falcão JP: Evaluation of four molecular typing methodologies as tools for determining taxonomy relations and for identifying species among Yersinia isolates. J Microbiol Methods 2010, 82:141-50.

28. Souza RA, Falcao DP, Falcao JP: Emended description of the species Yersinia massiliensis. Int J Syst Evol Microbiol 2010

29. Pourcel C, André-Mazeaud F, Neubauer H, Ramise F, Vergnaud G: Tandem repeats analysis for the high resolution phylogenetic analysis of Yersinia pestis. BMC Microbiol 2004, 4:22.

30. Denoeud F, Vergnaud G: Identification of polymorphic tandem repeats by direct comparison of genome sequence from different bacterial strains: a web-based resource. BMC Bioinformatics 2004, 5:4.
31. Li Y, Cu Y, Hauck Y, Platonov ME, Dai E, Song Y, Guo Z, Pourcel C, Dentovskaya SV, Anisimov AP, Yang R, Vergnaud G: Genotyping and phylogenetic analysis of Yersinia pestis by MLVA: insights into the worldwide expansion of Central Asia plague foci. PLOS ONE 2009, 4:e6000

32. Vogler AJ, Driebe EM, Lee J, Auerbach RK, Allender CJ, Stanley M, Kubota K, Andersen GL, Radnedge L, Worsham PL, Keim P, Wagner DM: Assays for the rapid and specific identification of North American Yersinia pestis and the common laboratory strain CO92. BioTech 2008, 44:201-205.

33. Sauer S, Kliem M: Mass spectrometry tools for the classification and identification of bacteria. Nat Rev Microbiol 2010, 8:74-82.

34. Lasch P, Nattermann H, Erhard M, Stmmler M, Grunow R, Bannert N, Appel B, Naumann D: MALDI-TOF mass spectrometry compatible inactivation method for highly pathogenic microbial cells and spores. Anal Chem 2008, 80:2026-2034

35. Tomaso H, Thullier P, Seibold E, Guglielmo V, Buckendahl A, Rahalison L, Neubauer H, Scholz HC, Splettstoesser WD: Comparison of hand-held test kits, immunofluorescence microscopy, enzyme-linked immunosorbent assay, and fow cytometric analysis for rapid presumptive identification of Yersinia pestis. J Clin Microbiol 2007, 45:3404-3407.

36. Elhanany E, Barak R, Fisher M, Kobiler D, Altboum Z: Detection of specific Bacillus anthracis spore biomarkers by matrix-assisted laser desorption/ ionization time-of-flight mass spectrometry. Rapid Commun Mass Spectrom 2001, 15:2110-2116.

37. Castanha ER, Fox A, Fox KF: Rapid discrimination of Bacillus anthracis from other members of the $B$. cereus group by mass and sequence of "intact" small acid soluble proteins (SASPs) using mass spectrometry. J Microbiol Methods 2006, 67:230-240.

doi:10.1186/1471-2180-10-285

Cite this article as: Ayyadurai et al:: Rapid identification and typing of Yersinia pestis and other Yersinia species by matrix-assisted laser desorption/ionization time-of-flight (MALDI-TOF) mass spectrometry. BMC Microbiology 2010 10:285.

\section{Submit your next manuscript to BioMed Central and take full advantage of:}

- Convenient online submission

- Thorough peer review

- No space constraints or color figure charges

- Immediate publication on acceptance

- Inclusion in PubMed, CAS, Scopus and Google Scholar

- Research which is freely available for redistribution
C Biomed Central 